\title{
Development of a Rehabilitation Mobility Assistive Device
}

\author{
Xueqiao Han, Reza Fotouhi*, Doug Bitner \\ Department of Mechanical Engineering \\ University of Saskatchewan \\ Saskatoon, Canada \\ * Corresponding author Reza.fotouhi@usask.ca
}

\begin{abstract}
- the aim of the overall research presented here was to investigate the motion of a wheeled mobile robot in an indoor (structured) setting while following a pre-set trajectory. An example of an application for this research would be automated maneuvering of a smart power wheelchair in a health care setting, such as a hospital, which would benefit the aging Canadian population. For the specific research reported here, the aim was to investigate the mobility of an assistive device for Sit-to-Stand (STS) operation and walking. A rehab robot was developed and was attached to a wheeled mobile robot to accomplish STS operation and to help walk a patient for rehabilitation. Four major phases of this research are: (i) design of the rehab robot, (ii) development of the control algorithm, (iii) experimentation, and (iv) navigation of the mobile robot and the rehab walker (robot). This research project can be extended to lower limb rehabilitation and design of a smart walker. It intensively studied current research and projects, designed a rehab robot and four control algorithms to help people in both STS and walking processes. Experiments were implemented, and the results indicated the effectiveness of the control algorithms and the prescribed navigation algorithm. According to the results, this project achieved the original goals to assist people in standing up and walking, and to navigate to a pre-set location.
\end{abstract}

Keywords: mobility assistive device; rehab robot; navigation

\section{BACKGROUND AND MOTIVATION}

As the aging population increases, more and more countries require a larger workforce to take care of the elderly. Meanwhile, the gradually soaring cost and investment in healthcare and nursing have become a global issue. Taking care of people with impaired lower limb functions is one of the trickiest problems. A number of elderly have problems with their knees, hips, and lower limb strength, which affects their mobility. They have difficulties in walking and are not able to cope well at home. People recovering from stroke, lower limb fracture, and some other illnesses suffer similar problems as well. Rehabilitation services and nursing are available, but access is limited due to constrained medical resources and their high cost. As a result, some of these people miss the best time for recovery, and their limited activity keeps them in their home and hence, they suffer from more health issues caused by lack of movement and being alone. One of the motivations for this research is to provide easy standing and walking assistance for these people and an alternative solution for nursing care as well. This is also expected to help in lower limb rehabilitation and patients' autonomy.

A variety of walkers are available on the market that help in walking, such as the rollator walker and the adjustable standard walker [15]. However, most traditional walkers are designed for people with a high self-care ability level. For people with poor health conditions, such as weakened leg strength, limited hand and finger motion, and hemiplegia sequelae, merely operating the walker would be a huge challenge. Traditional walkers do not provide support for Sitto-Stand (STS), which is one of the toughest challenges for self-autonomy. Hence, it leads to a second motivation for this research, to design a device that helps a broader range of people.

Motivated by medical needs as stated above, this project is focused on research of lower limb rehabilitation and assistance by devising a machine with the following capabilities:

1. Have basic walker functions.

2. Help in the STS process (the major goal of this design).

3. Provide assistance in standing and walking.

4. Be smart in navigation (this feature will provide care, convenience, and assistance to users, and allow patients for rehabilitation by walking in a pre-defined path).

5. Have a good level of adaptability (it should be able to help a wide range of people having different height, weight and health conditions, and be useful for home/hospital).

\section{A. Literature Review}

Several researchers proposed interesting designs and ideas, and also studied some related topics in this area.

Morbi et al. [1] designed a system called Gait-Enable, which is used in over the ground walking and balance training. This design integrated the Bungee Mobility Trainer, which is a patented elastic passive body weight support system that 
consists of a 3-DOF linkage that supports the patients' pelvis from below. The test results show that the Gait-Enable system will reduce pelvis and torso motion constraints. Ahmed et al. [2] designed an assistive device named E-Just Assistive Device (EJAD), a low cost intelligent assistive device to help elders in walking and sit-to-stand activities. This is a mechanical design based on the imitation of the caregivers' motion during the support task, which is mainly composed of back support, a robot arm and an active walker. Bauzel et al. [4, 5] analyzed current research on smart walkers and proposed three required significant features: provide dynamic support, demand little or no effort to use, be user-friendly. Also, a new user interface which allows users to control the walker intuitively was designed and implemented on a ASBGO walker, which is an active robotic walker that assists the elderly and disabled people in walking. Measures have been adopted to assure safety and customer comfort, such as infrared rays gesture detection, force detection of falls, and limit wheels' orientation and speed. Bulea et al. [6] proposed a new design with adapted gas spring actuators to enable auto lifting, utilizing both handles and platforms for arm support. The gas spring provides lifting forces and a steel shaft is designed to help users moving freely. The experimental results from a partially paralyzed male show that the lift walker significantly shortened the transition time of the sit-to-stand process and also resulted in a steadier rise than with a standard walker. Jorge et. al. [7] reviewed all current robotic systems for lower-limb rehabilitation in 2011 and pointed out that research in this field has grown exponentially during the last decade. About 43 robotic systems were mentioned that can be classified into the following five categories: Treadmill Gait Trainers, Foot-Plate-Based Gait Trainers, Overground Gait Trainers, Stationary Gait Trainers and Ankle and Knee Rehabilitations Systems made up of Stationary Systems and Active Foot Orthoses. Of these, more than half of the systems have neither been marketed nor applied for home use. This is due to their bulky size, elevated cost and the lack of high clinical improvement evidence. Research specifically in the STS area has also been investigated and has further contributed to this work. It has helped in developing and designing the motion control algorithms of the walker used in this project.

Other research work has been carried out to help the development of smart robotic walkers. Pasqui et. al. [8] found that the global shape of a patient's trajectory is an "s-like" curve. This was discovered through experiments participated by patients. Wang et al. [9] presented a fuzzy controller design for a lower limb rehabilitation robot, which is more effective than a traditional PID controller. Pasqui et al. [10] proposed a method to assess the efficiency of smart-walkers by measuring the time cost of different walkers when users were required to complete a series of movements. Other types of robotics to assist walking and lower limb rehabilitation have also been studied these include a new design of an exoskeleton system presented by Castro et al. [11], a new robot design for children with cerebral palsy presented by Giergiel et al. [12] and a mechanism model of a lower limb passive rehabilitation system built by Zhang et al. [13]. In addition, some researchers have also studied the psychological aspects of lower limb rehabilitation. For example, Koenig et al. [14] discussed a psychological measurement method which may help in robotic-assisted gait rehabilitation.

To summarize, some of the work done in this area has been reported here. However, most of the existing rehabilitation systems are not good enough for commercialization. Most of them have issues in cost, safety and portability; and also lack reliable testing. Unfortunately, none of the intelligent devices available can help both STS and the walking process. Hence, a mature, smart, and affordable rehabilitation system for STS and walking has yet to be designed.

\section{B. Research Objective}

The objectives of this research can be summarized as follows: 1- Investigate the state-of-the-art for the existence and needs of an assistive device for the sit-to-stand (STS) and walking operation. Develop such a device and its relevant motion control algorithms to help patients with rehabilitation.

2- Investigate and test the STS operation and the interaction between the user and the device. Test control algorithms for the rehab robot and evaluate its effectiveness in helping people with lower limbs rehabilitation

3- Investigate/examine navigation algorithms in a medical setting that may help in walking for rehabilitation.

\section{Research Methodology}

A literature review was performed and a device (rehab robot) was designed based on conclusions and ideas drawn from it. After a prototype of the rehab robot was manufactured, control algorithms for three different means of STS: arm-pad support, handlebar support, and belt support, were developed. Following this, practical tests with different control algorithms were implemented and analyzed. Also, a questionnaire was handed out to participants and the results were analyzed qualitatively. Lastly, the smart walker was attached to an Autonomous Ground Vehicle (AGV) which was used as the driver, and an existing navigation algorithm was tested to assess its feasibility for this project.

\section{DESIGN OF THE REHAB ROBOT}

Since this project is an attempt to develop an affordable mature smart walker, the AGV was adopted as a driver in the experiments. A rehab robot was designed to attach to the AGV and complete the major functions of a smart walker. This rehab robot has basic walker functions and adjusts its height according to a control algorithm or a user's commands, hence it helps in the STS process and walking.

There are three main parts in the design process: function analysis, mechanical design, and electrical design. Function analysis was applied first in order to guide both the mechanical and electrical design. Six major functions were determined as follows: help the STS process, be capable of moving, be automatic, be safe, be comfortable to various 
users, and be cost effective. Each function was also analyzed in detail [16], which assured that the design met the requirements.

After function analysis, a mechanical prototype was designed since it not only was needed to meet all requirements, but also had to provide sufficient convenience for electrical design and its functions. For example, a battery box was designed for the storage of batteries and other electrical components. There are several parts in the mechanical design section: conceptual design, detailed design, Finite Element Analysis and material selection. The rehab robot prototype is shown in Figure 1.

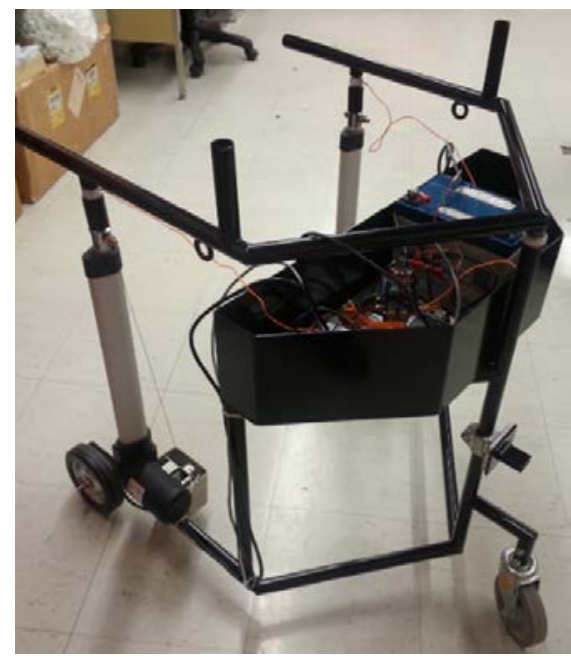

Figure 1. Prototype of the Rehab Robot

The electrical component design was developed for motion and control. First, the goal and requirements for the electrical design were clarified and then appropriate electrical components were selected (after extensive research). The electrical flowchart of the system is shown in Fig. 2. The basic idea is that microcontrollers (two Arduino Mega 2560) collect data from real-time transducers including, force sensors, position sensors, keypad inputs and as options, a sound detector and voice recognition module. Then the microcontrollers process the data through a programmed control algorithm downloaded to the microcontrollers and output the corresponding signals to a motor controller. Next, the motor controller, that uses a constant power supply from batteries and a voltage regulator, outputs a voltage that governs the motion of the actuators. Transducers collect data at one second intervals. Hence, the closed-loop control algorithm is able to control the motion of the actuators quickly and effectively.

Transducers in the prototype collect position and force data that mainly helps in detecting the users' status, such as sitting, standing, walking and falling. Keypad, screen, voice recognition module, sound detector, and speaker were designed as the user interface. This enabled the rehab robot to take in data that reflects user information and preference, and to react to real-time commands.

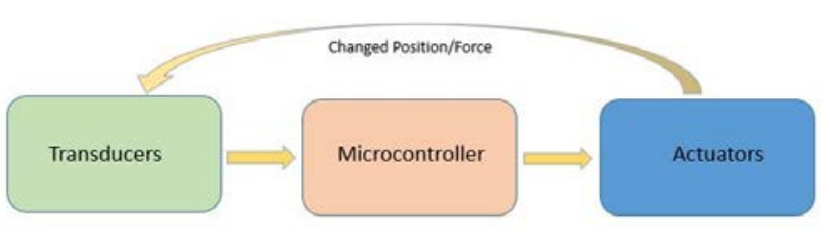

Figure 2. Electronics Connection Schematic Diagram

\section{CONTROL ALGORITHM DESIGN AND RESEARCH}

The algorithms developed in this project utilized three different techniques to help in the STS process: 1-arm-pad support, 2-handlebar support, and 3-belt support, as shown in Fig. 3. There are two algorithms (Test 3 and Test 2-1) for armpad support, one algorithm (Test 4-2) for handlebar support, and one for belt support (Test 5). More algorithms and corresponding test information can be found in Table 1 .

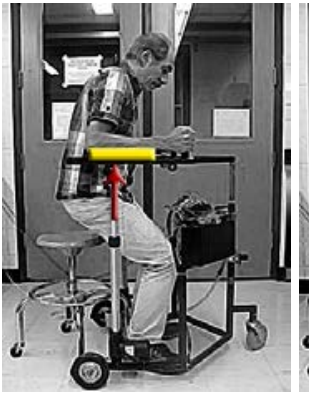

(a) Arm-pad Support

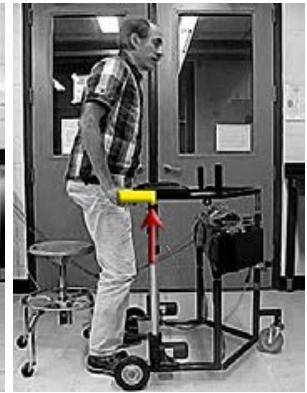

(b) Handlebar Support

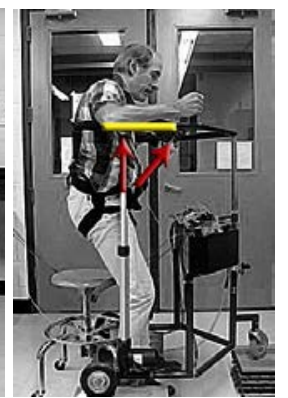

(c) Belt Support
Figure 3. Three Lifting Methods

These algorithms were used to first detect the status of the user and then react appropriately for each status. For the arm-pad support algorithms, once the device detects a user, it triggers the upper frame with the support arm-pad to lift to a theoretical best height, calculated from the applied force on the actuators, user's height and weight. Once it reaches the theoretical height, it enters into a height adjustment phase, where the user will be able to adjust the height automatically or manually. When the adjusting phase is completed, the rehab robot goes into a steady state mode and maintains the same height for the upcoming walking process. When no input is received from the user after a specified period, the actuators will retract to the initial position and wait for the next round of usage. The schematic diagram of the control algorithm is shown in Fig. 4.

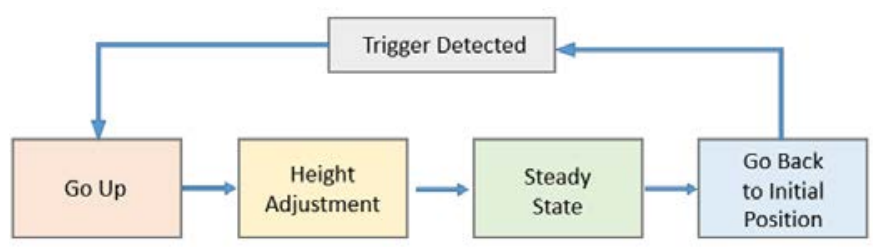

Figure 4. General Control Algorithm Concept Diagram 
The belt support algorithm is very similar to the arm-pad support algorithm, except that the actuators are lifted to a higher position first to enable the belt to help lift the user to a fully upright position. It will then be lowered to the theoretical best height before entering into the height adjustment phase.

The handlebar support algorithm is a simple algorithm that controls the height of the rehab robot to follow the trajectory of the desired hand height of the user during the STS process. Abnormal cases have been considered in all algorithms, and voice control is also available. A flowchart for each algorithm and a detailed introduction to these can be found in section 3.1 of [16].

\section{RESULTS AND ANALYSIS}

This section discusses the test process for the control algorithms, provides results, analysis and comparison with all experiments conducted. Seven experiments were conducted with the help of three participants. Table 1 shows a summary of these experiments. In general, the tests demonstrated in [16, Chapter 4] that they can also be categorized into three classes: arm-pad support lift tests (Test 1-1, Test 1-2, Test 2-1, Test 3), handlebar support lift tests (Test 2-2, Test 4-2), and belt support lift test (Test 5). Also Natural STS is Test 4-1.

TABLE 1. TEST List of ReHAB RoBot (ALL ALGORITHMS USE FORCE AND DISPLACEMENT FEEDBACK)

\begin{tabular}{|c|c|c|c|}
\hline Test No. & Description & Controller Type & Importance \\
\hline Test 1-1 & ift the upper frame to preset practical best height for a particular use & Open Loop & Secondary \\
\hline Test 1-2 & Lift the upper frame to highest position & Open Loop & Secondary \\
\hline Test 2-1 & Controlled by Applied Force Based Arm-pad Lifting Methond & Closed Loop & Primary \\
\hline Test 2-2 & Controlled by Simple Handlebar Lifting Method & Closed Loop & Secondary \\
\hline Test 3 & Controlled by Body Weight Based Arm-pad Lifting Methond & Closed Loop & Primary \\
\hline Test 4-1 & Natural STS process & No Device Required & Secondary \\
\hline Test 4-2 & Lift the handlebar to preset practical best height for a particular user & Open Loop & Secondary \\
\hline Test 5 & Controlled by Belt Support Lifing Method & Closed Loop & Primary \\
\hline
\end{tabular}

The purpose of discussing the eight tests in this project is to compare the three different support lifting methods, and also compare the effectiveness of the different algorithms for the same lifting method. In general, all three lifting methods provide assistance to the STS process, and require less leg strength, compared with the natural STS process. The belt support lifting method provides the most assistance, which can be concluded from Comparison I: Arm Force Comparison [16, section 4.4]. As the force on arm settles down quicker than other approaches. The other two lifting methods provide a similar amount of support assistance during the STS process. Fig. 5 is a typical result of arm force comparison for different algorithms. For overall lower limb assistance, the arm-pad support lifting method could be the best, because it also carries the upper limb weight during the walking process, and maybe some upper trunk weight as well. The results can be found in detail in Comparison II: Leg Force Comparison [16, section 4.6]. Fig. 6 shows the averaged test results comparison.

In the two control algorithms designed for the arm-pad support lifting method, the Applied Force Based control algorithm was found to be the better algorithm, according to Comparison I: Arm Force Comparison [16, section 4.4]. Perhaps, this is because this algorithm is more stable, and it takes less time for the participants to find their best arm height.

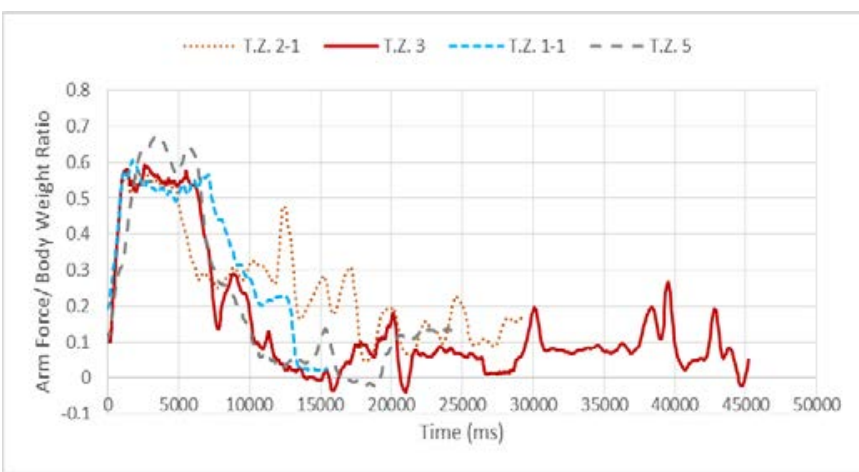

Figure 5. Test Results of the Participant TZ (Force on Arms Comparison), arm-pad support (Tests, 1-1, 2-1, 3), belt support (Test 5).

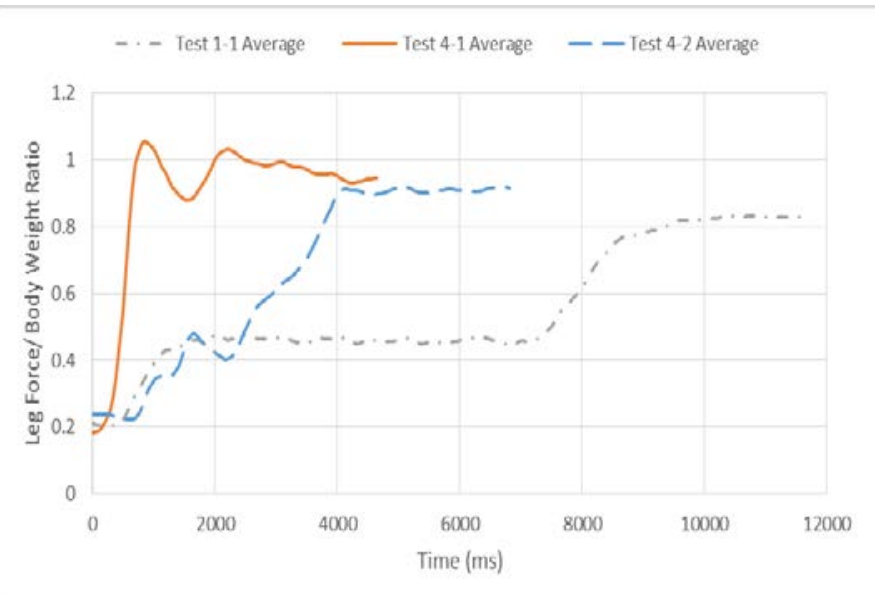

Figure 6. Comparing Different Methods of STS (Leg Force), average results of three participants. Arm-pad support (Test 1-1), Natural STS (Test 4-1), Handlebar support (Test 4-2).

\section{NAVIGATION ALGORITHM AND TESTS}

To demonstrate the walking aspect of the rehab robot, a Fuzzy-Logic-Based (FLB) algorithm [18] was adopted for navigation of the $\mathrm{AGV}$ driving the rehab robot in an indoor medical setting. Using this algorithm, the AGV was able to successfully reach several goals while avoiding obstacles during its motion.

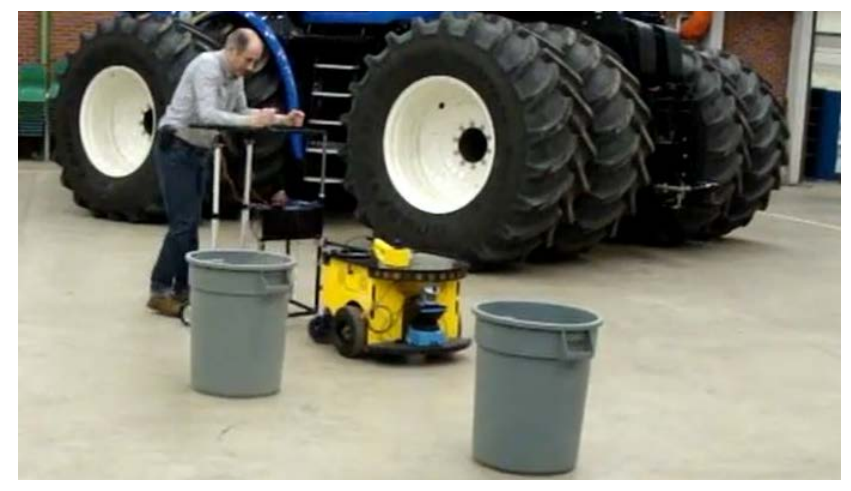

Figure 7. Test of rehab-robot Avoiding Obstacles 
The rehab robot was attached to the AGV and tested using the FLB algorithm (see Fig. 7). Based on H. Barden's gait speed classification [17], three speed tests were implemented: 0.9 $\mathrm{m} / \mathrm{s}, 0.65 \mathrm{~m} / \mathrm{s}, 0.4 \mathrm{~m} / \mathrm{s}$, and one-obstacle and two-obstacle cases were tested for each speed. During the tests, the device successfully reached preassigned goals and avoided obstacles, however, it made sharp turns, and the user experience was not ideal for practical applications. Fig. 8 shows a typical example of navigation of the rehab robot while avoiding a two-obstacle situation. Detailed test results and findings can be found in [16, section 5.3].

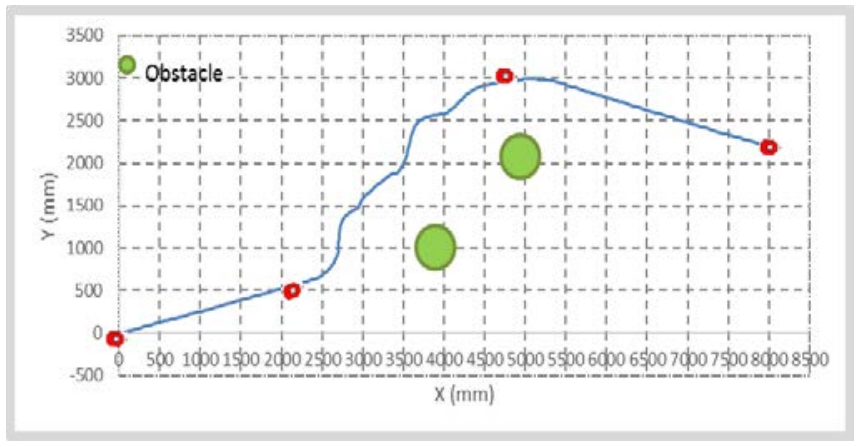

Figure 8. Typical Test Results of Avoiding Two Obstacles (Path). (X: Horizontal Displacement, Y: Vertical Displacement, Goal Position: (8000, 2200))

\section{CONCLUSIONS}

The design of the mechanical and electrical parts, development and comparison of the algorithms, and tests of the navigation algorithm were addressed here. Also, several participants tested the algorithms. The results showed that the designed algorithms helped in the STS process and aided users in finding a comfortable walking position within a reasonable time. According to the results of experiments and questionnaires, the four developed algorithms successfully helped participants to complete the STS process and provided a relatively good user experience at the same time. Hence, it can be concluded that major design goals for the rehab robot have been achieved. However, for future research and possible commercialization, some design details have to be improved and large-scale test data is needed for optimizing the control algorithm. After the rehab robot was fully developed, it was attached to a wheeled mobile robot and went through a set of tests. For navigation, the FLB algorithm adopted in this project, helped in reaching the goal position while avoiding obstacles. However, the user experience was not ideal and hence the navigation aspect of this research needs to be modified for use in any future medical applications.

\section{REFERENCES}

[1] Morbi, A.; Ahmadi, M.; Nativ, A., GaitEnable: An omnidirectional robotic system for gait rehabilitation, Mechatronics and Automation (ICMA) International Conference, pp.936,941, 5-8 Aug. 2012.
[2] Koenig, S; Omlin, X; Zimmerli, L; Sapa, M; Krewer, C; Bolliger, M; Müller, F; Riener, R.; A Systematic Approach for Design a Low-Cost Mobility Assistive Device for Elderly People; Int. Journal of Medical, Health, Biomedical and Pharmaceutical Engineering; Vol.5, No.11, pp.597,602; 2011.

[3] Omar Salah, Ahmed A. Ramadan, Salvatore Sessa, Ahmed Abo Ismail, Makasatsu Fujie, Atsuo Takanishi. ANFIS-based Sensor Fusion System of Sit-to-stand for Elderly People Assistive Device Protocols. International Journal of Automation and Computing, vol. 10, no. 5, pp. 405-413, 2013.

[4] Martins, M.; Santos, C.; Seabra, E.; Frizera, A.; Ceres, R., "Design, implementation and testing of a new user interface for a smart walker," 2014 IEEE International Conference on Autonomous Robot Systems and Competitions (ICARSC), Espinho, pp.217,222, 14-15 May 2014.

[5] Martinsa M, Santosa C, Frizerab A, Ceresc R. Real time control of the ASBGo walker through a physical human-robot interface. Measurement. Vol.48, No. 2, pp.77, 86. 2014.

[6] Bulea T,Triolo R. Design and Experimental Evaluation of a Vertical Lift Walker for Sit-to-Stand Transition Assistance. Journal of Medical Devices. Vol.6, Issue 1, 2012.

[7] Díaz I, Gil J, Sánchez E. Lower-Limb Robotic Rehabilitation: Literature Review and Challenges. Journal of Robotics. Vol.2011, 11 pages, 2011

[8] Pasqui, V; Saint-Bauzel L; Sigaud O. Characterization of a Least Effort User-Centered Trajectory for Sit-to-Stand Assistance. In: Stephan G; Kovacs L; Toth A IUTAM Symposium on Dynamics Modeling and Interaction Control in Virtual and Real Environments. Springer Netherlands, Vol. 30, pp197-204, 2011.

[9] Hongche Guo; Ziming Wang, "Design of Fuzzy Controller Used in Lower Limbs Rehabilitative Robot Drive System Based on Genetic Algorithm," Fuzzy Systems and Knowledge Discovery, Fourth International Conference on, vol.4, pp.168,171, 24-27 Aug. 2007.

[10] Pasqui V; Saint-Bauzel L; Rumeau P; Vigouroux N. Proposed Generic Method to Assess Efficiency of Smart-Walkers. Lecture Notes in Computer Science. Vol. 7251, pp 218-221, 2012.

[11] Castro, S.; Lugo, E.; Ponce, P.; Molina, A., "Assistive Robotic Exoskeleton for Helping Limb Girdle Muscular Dystrophy," Mechatronics, International Conference on Electronics and Automotive Engineering (ICMEAE), Morelos, pp.27, 32, 19-22 Nov. 2013.

[12] Mariusz Giergiel, Aleksander Budziński, Grzegorz Piątek, Michał Wacławski. Personal Lower Limb Rehabilitation Robot for Children. In: Jan Awrejcewicz, Roman Szewczyk, Maciej Trojnacki ,Małgorzata Kaliczyńska Mechatronics - Ideas for Industrial Application. Springer International Publishing, 169-176, 2015.

[13] Zhang M; Li X, "A Design of the Mechanism for Lower Limbs Passive Rehabilitation and Kinematics Analysis," 2010 Fifth International Conference on Frontier of Computer Science and Technology (FCST), Changchun, Jilin Province, pp.603,607, 18-22 Aug. 2010.

[14] Koenig, S;Omlin X;Zimmerli L; Sapa M;Krewer C;Bolliger M; Müller F; Riener R. Psychological state estimation from physiological recordings during robot-assisted gait rehabilitation. Journal of Rehabilitation Research \& Development. Vol. 48, 367-386, 2011.

[15] Drive Medical, Four Wheel Walker Rollator with Fold Up Removable Back Support, 2017. https://www.amazon.ca/Wheel-Walker-RollatorRemovable-Support/dp/B005S1CHKC/, accessed Apr 22, 2017.

[16] Han, X., Development of a Mobility Assistive Device for Sit-to-Stand, M.Sc. thesis, Department of Mechanical Engineering, University of Saskatchewan, Saskatoon, Canada, 2017.

[17] Heather Braden, Self-selected gait speed: A critical clinical outcome, 2012. http://lermagazine.com/article/self-selected-gait-speed-a-criticalclinical-outcome, accessed May 26, 2017.

[18] Heidari, F., Vakil, M., Fotouhi, R., Sensor-based navigation of agricultural autonomous mobile robots, ASME Int. Design Engineering Technical Conf, Chicago, USA, Aug 12-15, DETC2012-71348, 2012. 